\title{
全球变化对食物网结构影响机制的研究进展
}

\author{
王晴晴 高 燕 王 嵘* \\ 华东师范大学生态与环境科学学院, 浙江天童森林生态系统国家野外科学观测研究站, 上海 200241
}

\begin{abstract}
摘 要 食物网主要依靠基于不同营养级间物种互作形成的上行与下行调控维持其结构。全球变化能够改变种间关系，威胁 生物多样性的维持, 然而目前对全球变化改变食物网结构的机制仍处于探索阶段。近年来通过大时空格局与多营养级食物网 研究, 发现全球变化的作用机制主要可归结为 3 种: 物候错配、关键种丧失与生物入侵。该文聚焦于这 3 种机制, 综述各种机 制造成的食物网结构变化并探讨相关的进化与生态驱动因素。三种干扰机制均通过改变原有种间关系, 影响食物网调控, 改 变食物网结构。不同的是, 物候错配造成的种间关系变化是由于不同物种的物候对全球变化产生非同步响应所致; 关键种丧 失则使营养级间取食/捕食关系发生变化甚至缺失; 而入侵物种通过竞争排除同营养级物种改变种间关系。最后, 该文提出食 物网结构变化的实质是物种是否能够适应快速变化的生态环境, 并据此展望未来研究方向。随着全球变化影响日益加剧, 急 需继续深入探索导致全球变化下食物网结构改变的机制, 为制定合理的生物多样性保护与生态修复规划提供重要理论支撑。 关键词 全球变化; 种间关系; 食物网结构; 物候错配; 关键种丧失; 生物入侵
\end{abstract}

王晴晴, 高燕, 王嵘 (2021). 全球变化对食物网结构影响机制的研究进展. 植物生态学报, 45, 1064-1074. DOI: 10.17521/cjpe.2020.0061

\section{Review on impacts of global change on food web structure}

\author{
WANG Qing-Qing, GAO Yan, and WANG Rong* \\ Zhejiang Tiantong Forest Ecosystem National Observation and Research Station, School of Ecological and Environmental Sciences, East China Normal Uni- \\ versity, Shanghai 200241, China
}

\begin{abstract}
The food web sustains its structure mainly by bottom-up and top-down regulations of the species interactions among different trophic levels. However, global changes can alter interspecific relationships and threaten the maintenance of biodiversity. It is still unclear how global change alters the structure of the food webs. In recent years, based on numerous studies on food webs composed of multi-trophic levels at large spatiotemporal scales, researchers have found that global changes alter food web structure mainly through three mechanisms: phenological mismatching, loss of key species and biological invasion. Here we focused on these three mechanisms and reviewed how these mechanisms regulate food web structure change, with further discussions on the driving factors in ecology and evolution. All these three mechanisms can alter the interspecific interactions, resulting in distortion of the regulation of food webs. The major difference among these three mechanisms is how interspecific interactions are changed. Phenological mismatching occurs due to the asynchronous responses in the phenology of different species to global changes, while the loss of key species can change or even entirely destroy some critical feeding/predation relationships, and invasive species often simplify the food web structure by causing strong interspecific competition to exclude species at the same trophic level. Finally, we pointed out that the changes in food web structure actually depend on the adaptation of species to the ongoing global changes and we further provided some insights into future research directions. With aggravated global change impacts, it is necessary to further study the mechanisms underlying how global changes influence food web structure, to reinforce the extant theoretical basis for formulating biodiversity conservation and ecological restoration measures.

Key words global change; interspecific interactions; food web structure; phenological mismatching; loss of keystone species; biological invasion
\end{abstract}

Wang QQ, Gao Y, Wang R (2021). Review on impacts of global change on food web structure. Chinese Journal of Plant Ecology, 45, 1064-1074. DOI: 10.17521/cjpe.2020.0061 
食物网是指由个体、种群与营养级之间形成的 生物间取食关系网络，也称为“消费者一资源”互作 网络, 广义上认为除不同营养级间物种的拮抗关系 外也考虑种间互惠关系以及同一营养级中物种的竞 争关系(李妍等, 2008; Wabnitz et al., 2010)。食物网 概念是基于Charles Elton在1927年提出的食物链理 论发展而来的(Elton, 1958), 通过Raymond Lindeman 和Robert May等生态学家的不断发展形成了系统的 食物网理论 (Lindeman, 1942; May, 1972; Pimm, 1979), 并在21世纪初将复杂网络理论引入食物网 研究中(Dunne et al., 2002), 通过节点和路径的分布 特征描述了系统的连通性和最优性, 为食物网研究 提供了全新的理论框架, 促进食物网研究在近 20 年 中快速发展。近年来, 我国学者在食物网相关研究 领域已取得重要进展, 结合食物网结构与生物多样 性, 研究垂直多样性和水平多样性以及时空尺度对 食物网动态和稳定性的影响(Wang \& Brose, 2018; Wang et al., 2019c)。

目前主要认为食物网主要依靠上行(bottom-up) 与下行(top-down)调控来维持其结构、生物多样性与 生态系统功能。Lindeman (1942)首次定量研究了能 量在不同营养级之间的传递过程, 强调了食物网动 态的上行调控机制。20世纪60年代, Hairston等美国 生态学家提出的“绿色世界假说” (green world hypothesis), 强调捕食者对食草动物和植物的下行调 控作用(Hairston et al., 1960)。上行与下行调控共存 于绝大部分生态系统中, 两种调控共同作用从而限 制各营养级中所有物种的种群大小是促使物种共 存、维持生物多样性的重要机制(Terborgh et al., 2001; Ewers \& Didham, 2006; Estes et al., 2011; Wang et al., 2019b, 2020)。

在人类世(Anthropocene)中人类活动造成了包 括全球气候快速变化、生境破碎化与环境污染等一 系列全球变化问题, 已在全球范围内造成了严重的 生态后果(Tylianakis et al., 2008; Butchart et al., 2010)。例如, 在全球气温与降水量迅速变化的背景 下, 众多物种的自然分布范围正发生巨大变化, 甚 至灭绝(Gilman et al., 2010); 同时世界上几乎所有 的森林生态系统均发生了片段化现象, 导致动植物 栖息地大量丧失, 并引发大型猎食动物种类与数量 快速下降(Taubert et al., 2018); 此外, 全球范围内频 繁的人类活动例如国际贸易促使大量外来物种入侵,
直接改变入侵地生态系统中的物种组成与群落结构 (Simberloff et al., 2013)。有研究估计人类活动已导 致全球物种数量下降了 $18.3 \%$ (Murphy \& Romanuk, 2014)。

全球变化可能直接使物种灭绝(Butchart et al., 2010; Cardinale et al., 2012), 也可能通过改变种间 关系, 影响食物网结构(Memmott et al., 2004; Bascompte, 2009; 王玉玉等, 2013; Ripple et al., 2014)。 然而, 种间关系的改变影响上行与下行效应进而改 变食物网结构的潜在机制的研究仍处于探索阶段。 近年来通过众多大时空尺度和多营养级食物网研究, 对这一问题有了一定的认识, 可大体归结为 3 种机 制: 物候错配、关键种丧失与生物入侵。本文将聚 焦于全球变化影响食物网结构这一主题, 介绍上述 3 种机制造成食物网上行与下行调控变化的过程与 后果, 据此展望未来生物多样性保护与生态修复的 研究方向。

\section{1 全球变化影响食物网结构的机制}

\section{1 物候错配}

食物网中互作物种的物候特征(例如植物的开 花物候与传粉动物的活跃期和繁殖期; 浮游生物的 种群峰值与鱼类的繁殖期等)往往存在时空耦合 (Gilman et al., 2010)。然而，由于不同物种的物候特 征对全球变化的响应存在巨大差异, 使得原本耦合 的关系发生错配, 即物候错配。物候错配改变了不 同营养级物种的相互作用强度, 甚至消除这一关系, 阻碍能量由低营养级向高营养级流动, 导致高营养 级物种多度下降甚至灭绝, 进而降低食物链长度与 食物网复杂性(Gilman et al., 2010; Sydeman et al., 2015)。

目前普遍认为全球气候变暖是导致物候特征快 速变化的主要驱动因素。Thackeray等(2016)通过综 合分析 10003 个涵盖 812 种海洋、淡水和陆地物种的 长期物候观察记录(超过20年), 发现几乎所有物种 的重要物候特征发生期均随着时间推移而提前, 但 不同营养级物种提前的幅度不一样, 其中初级消费 者物候特征发生期提前的时间最长, 平均达 6.2 天, 这种非同步的物候特征变化促使物候错配的发生。 现有研究已找到了物候特征变化影响不同营养级间 种间关系的证据, 例如全球范围内传粉者多度日益 下降也与气候变暖有关。植物在变暖环境中提前开 
花, 使传粉昆虫活跃期与花期重叠时间减少, 导致 动植物群落衰退(Gilman et al., 2010; Potts et al., 2010)。气候变化驱动的物候特征改变也已在一些特 殊生态系统中被发现。通过采用内耳石测量法分析 青藏高原湖泊中的特有鱼类色林错裸鲤(Gymnocypris selincuoensis)的繁殖物候, 发现其与 20 世纪70 年代至 21 世纪初的气候变化有关, 该鱼类的繁殖期 平均每 10 年提前 2.9 天, 这一大型鱼类的物候特征变 化可能会影响本地水体生态系统的食物网结构及其 种群更新(Tao et al., 2018)。

物候错配不仅发生于生态系统内部, 不同生态 系统中物种的物候特征变化能够在更大尺度上造成 物候错配。1984-2004年间, 在荷兰分布的栖息于森 林生境的长距离迁徙食虫鸟类的种类急剧减少, 这 种现象的重要成因是由于全球变暖导致西欧的春天 变得更为温暖, 不同森林生态系统中的昆虫物候特 征也发生的快速变化致使鸟类食物资源供应峰值与 长距离迁徙食虫鸟类的到达与滞留时间不匹配 (Both et al., 2010)。

物候错配现象也在人工控制实验系统一一青藏 高原草甸中的常见植食昆虫夜蛾(Melanchra pisi)及 其宿主植物条叶银莲花 (Anemone trullifolia var. linearis)与龙胆属植物美丽龙胆(Gentiana formosa)中 得到了验证。通常夜蛾幼虫主要以条叶银莲花为食, 而美丽龙胆通常在夜蛾幼虫密度达到高峰后才开花, 从而避免花序被夜蛾幼虫过度啃食。然而, 在为期 3 年的人工增温实验中, 美丽龙胆花期和条叶银莲花 的营养繁殖物候在温度提升 $1.5{ }^{\circ} \mathrm{C}$ 的情况下提前了 一周, 而夜蛾幼虫的出现却推迟了两周; 在升温状 态下夜蛾幼虫密度增加了10倍, 但条叶银莲花的密 度却仅下降了 $30 \%$, 而物候特征变化使夜蛾幼虫主 要取食美丽龙胆的花和胚珠, 对美丽龙胆造成的损 伤增加了 100倍(Liu et al., 2011)。这种急剧加强的营 养级关联大大降低了美丽龙胆的繁殖能力, 因此气 候变暖导致的物候错配是导致植被衰退的潜在因素。

全球气候变化的另一个主要特征是昼夜温差加 大, 这一变化也能够导致物候错配。以瓶子草属植 物Sarracenia purpurea叶片中的水生微生物群落为 研究对象, 检验昼夜温差加大对不同微生物营养级 的效应, 结果表明与正常昼夜温差中的群落相比, 处于第一营养级的细菌密度在实验初始阶段增加后
开始迅速下降，而位于第二营养级的原生生物由于 生长节律与细菌群落动态错配造成食源匮乏, 导致 其 $\alpha$ 多样性急剧降低(Zander et al., 2017)。

除全球气候变化外, 许多其他类型的人类干扰 也能够改变区域气候。例如, 生境片段化将大生境 斑块分割为大量小生境片段, 导致边缘效应增强, 改变原有的林内小气候并改变物候特征, 可能导致 物候错配进而影响种间关系的稳定(Haddad et al., 2015; Wilson et al., 2016)。物候特征异步变化是导致 物候错配的主要因素。在受干扰环境中物候特征变 化速率可能与物种的世代周期有关, 世代周期越短 的生物适应环境变化的速度越快, 这与草本、灌木 和小型消费者(例如植食性昆虫)物候提前幅度较大 一致(Both et al., 2009; Thackeray et al., 2016)。目前 尚不清楚不同生物类群适应气候变化的潜在机制, 这可能涉及由温度变化引发激素分泌变化调整生长 节律以及表观遗传因子引发的可塑性变化。但是, 物种对气候变化的耐受能力是有极限的, Trisos等 (2020)研究了 30000 种陆地和海洋生物能够承受的 温度阈值并根据模型预测发现大量物种将由于全球 气温持续升高而在近期集体灭绝。然而, 在某些生态 系统中气候变化却未导致物候错配的发生(Hansson et al., 2013)。因此, 需要更多案例研究以全面分析 导致物候特征变化异步性的生物(例如种内遗传变 异水平)与环境因素(气候变暖程度、昼夜温差范围、 生境斑块大小等)并帮助预测未来不同生态系统中 食物网结构演变的趋势。此外, 地下生物群落尤其 是土壤微生物与小型土壤动物群落对生态系统服务 功能贡献巨大且对食物网结构具有重要的支持作用, 但由于物种鉴定困难等原因, 目前关于此类群落中 物候错配的报道较少。未来可结合DNA条形码(表1) 技术深入了解这些群落中的食物网结构与动态及其 对气候变化的响应。

\section{2 关键种丧失}

食物网中的关键种是对整个食物网的稳定具有 重要作用的物种, 这些物种的丧失能够产生强大的 营养级联效应，直接或间接导致大量物种灭绝 (Zhao et al., 2016)。关键种在食物网中往往处于连接 较多的节点上或相互作用强度高的连接节点上, 此 外, 能够对大量其他物种造成强烈间接影响的物种 (例如顶级捕食者)也是关键种。全球变化导致生态 
表1 文中相关专业术语解释

Table 1 Explanation of terminology in this review

\begin{tabular}{|c|c|}
\hline \multicolumn{2}{|c|}{ 专业术语 Terminology解释 Explanation } \\
\hline 上行调控 & 通过低营养级物种的防御限制高营养级物种的可利用食物资源进而调控食物网中各物种的种群大小(Terborgh et al., 2001)。 \\
\hline Bottom-up control & $\begin{array}{l}\text { The defenses of species at low trophic levels limit the availability of food resource for species at high trophic levels, regulating th } \\
\text { population size of each species in a food web (Terborgh et al., 2001). }\end{array}$ \\
\hline 下行调控 & 通过高营养级物种对低营养级物种的捕食/取食控制食物网中各物种种群大小(Terborgh et al., 2001)。 \\
\hline Top-down control & $\begin{array}{l}\text { Species at high trophic levels control the population size of each species in a food web through preying on or feeding on the species } \\
\text { low trophic levels (Terborgh et al., 2001). }\end{array}$ \\
\hline $\begin{array}{l}\text { DNA条形码技术 } \\
\text { DNA barcoding }\end{array}$ & $\begin{array}{l}\text { DNA条形码是指基因组中能够代表该物种的且在种间有足够变异的、易扩增的DNA片段。通过获取一个或多个DNA条形码片 } \\
\text { 段信息并与与数据库中相关序列进行对比可快速、精确地完成物种鉴定(Hebert et al., 2003)。 } \\
\text { The DNA fragments can represent the genomic characters of a species but with sufficient interspecific genetic variations and can be } \\
\text { easily amplified. Rapid and accurate species identification can be achieved by using one to several DNA barcode fragments and com- } \\
\text { paring the obtained sequence information with related sequences in gene databases (Hebert et al., 2003). }\end{array}$ \\
\hline \multirow[t]{2}{*}{$\begin{array}{l}\text { 进化军备竞赛 } \\
\text { Evolutionary arm race }\end{array}$} & $\begin{array}{l}\text { 自然选择在不断提高捕食者发现和捕获猎物效率的同时也会不断改进猎物及时发现和逃避捕食者的能力, 这种相互适应的过 } \\
\text { 化程被称为进化军备竞(You et al., 2013)。 }\end{array}$ \\
\hline & $\begin{array}{l}\text { As a result of reciprocal selection, the efficiency of predators in finding and capturing preys and the ability of preys in detecting and } \\
\text { eluding predators are simultaneously and continuously improved. This type of coadaptation in evolutionary history is named as the } \\
\text { evolutionary arms race (You et al., 2013). }\end{array}$ \\
\hline $\begin{array}{l}\text { 内禀优势 } \\
\text { Inherent superiority }\end{array}$ & $\begin{array}{l}\text { 外来种在繁殖和扩散的过程中, 某些固有特征(如生理、生态、遗传和行为等)相对于本地种具有竞争优势, 从而导致其成功入侵 } \\
\text { (Zou et al., 2007)。 } \\
\text { In reproduction and dispersal processes, many alien species have advantages compared with native species, due to their inherent charac- } \\
\text { teristics in some aspects like physiology, ecology, genetics, and behavior, consequently resulting in successful invasions (Zou et al., 2007). }\end{array}$ \\
\hline \multirow[t]{2}{*}{ 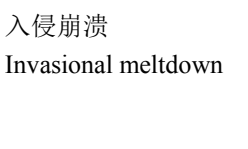 } & $\begin{array}{l}\text { 两个或多个外来物种间产生互惠关系, 促进它们在新生境中的种群建立、繁殖与扩散, 最终导致这些物种共同入侵(Ricciardi } 8 \\
\text { MacIsac, 2000)。 }\end{array}$ \\
\hline & $\begin{array}{l}\text { The population establishment, reproduction and dispersal of two or more alien species in the novel environments were facilitated by } \\
\text { their reciprocal mutualism(s), ultimately leading to the co-invasion of these species (Ricciardi \& MacIsaac, 2000). }\end{array}$ \\
\hline
\end{tabular}

系统中大量关键种丧失(Butchart et al., 2010; Wardle et al., 2011)。不同营养级中关键种丢失对食物网结 构造成的影响与作用机制存在较大差异。

顶级捕食者是被广为认可的关键种, 其缺失引 起生态系统下行调控机制丧失, 刺激消费者密度快 速增加从而加大对生产者的取食力度，抑制生产者， 最终导致整个食物网崩溃(图1)。Ripple等(2014)发现 包括狮子(Panthera leo)与灰狼(Canis lupus)在内的7 种大型肉食动物的消失对食物网中其他物种产生显 著影响, 部分物种在这些顶级捕食者丧失后多度变 化甚至超过100倍。顶级捕食者对食物网结构稳定性 的贡献也体现于受损生态系统的修复过程中。通过 恢复近岸生态系统中的顶级捕食者海獭(Enhydra lutris)的种群, 可以促进大叶藻(Zostera marina)海 草床的恢复(Hughes et al., 2013)。这是因为海獭能够 抑制蟹类对草食动物的捕食, 而草食动物增加使得 导致大叶藻窒息死亡的附生海藻的数量下降。频繁 的人类活动是造成顶级捕食者丧失的首要原因, 过 度狩猎往往能在短期内大大减少大型肉食动物的数 量, 同时全球范围内土地利用类型的改变缩小了顶 级捕食者的栖息地, 并隔离残存种群, 进一步威胁 到它们的生存(Ripple et al., 2014; Wilson et al., 2016)。此外, 全球气候变化引发的物候特征改变, 使长距离迁徙的食虫鸟类食源匮乏, 也是导致其种
群快速下降的重要因素(Both et al., 2010)。

某些消费者也是关键种，它们往往既以多种生 产者为食，也是众多捕食者的猎物。理论模型预测 消费者关键种的丧失, 可能阻碍营养级间的正常能 量流动，抑制捕食者的能量来源，影响捕食者多度 与食物网稳定性(Kadoya et al., 2018)。同时, 消费者 关键种丧失可能改变表观竞争格局，导致植物间竞 争加剧, 物种数减少, 降低食物网复杂性(图1)。传 粉者、种子传播动物与根瘤菌是另一类消费者关键 种, 它们与植物构成的互惠网络维持了植物种群的 繁殖与更新。这一物种类群的衰退已对生物多样性 与生态系统服务功能的维持构成严重威胁(Potts et al., 2010)。消费者关键种丧失与过度狩猎、生境 片段化与气候变化等干扰因素有关(Potts et al., 2010; Ripple et al., 2015)。

生产者是食物网的基础，对其上各营养级施加 上行调控效应(Scherber et al., 2010)。关键生产者的 丧失能够增强消费者取食其他生产者的力度, 造成 生产者大量灭绝，最终威胁消费者和顶级捕食者的 生存，导致食物网结构崩溃(图1)。群落中的优势植 物物种往往被认为是关键种。然而, 生产者中的稀 有物种也可能成为食物网中的关键种, 例如在岩质 海岸生态系统中, 将占岩石表面固着生产者生物量 小于 $10 \%$ 的海藻与无脊椎动物移除使消费者生物量 


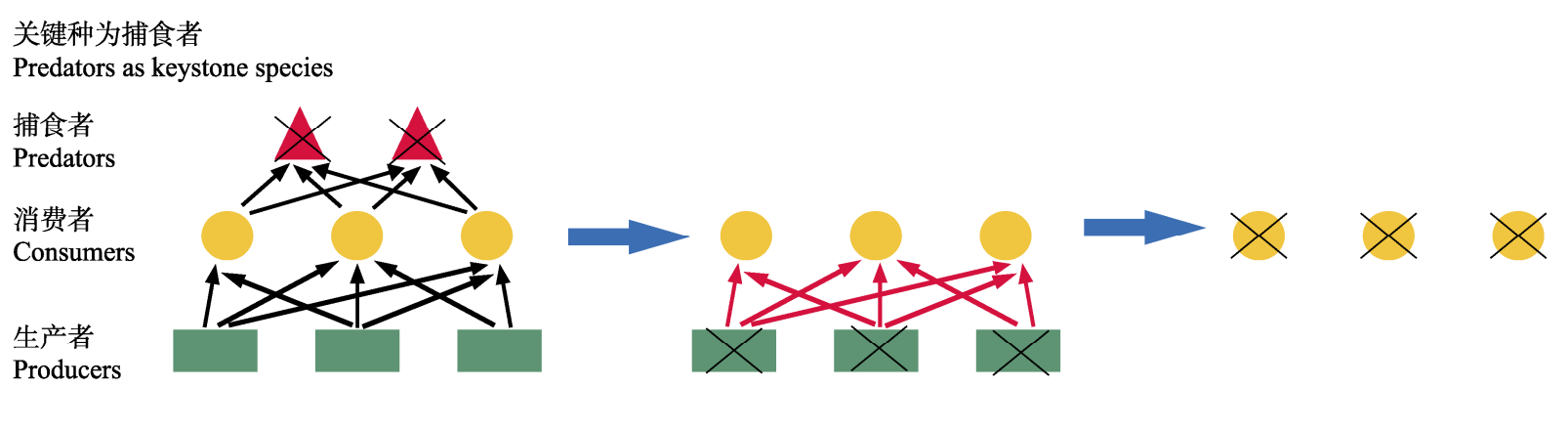

\section{关键种为消费者}

Consumers as keystone species

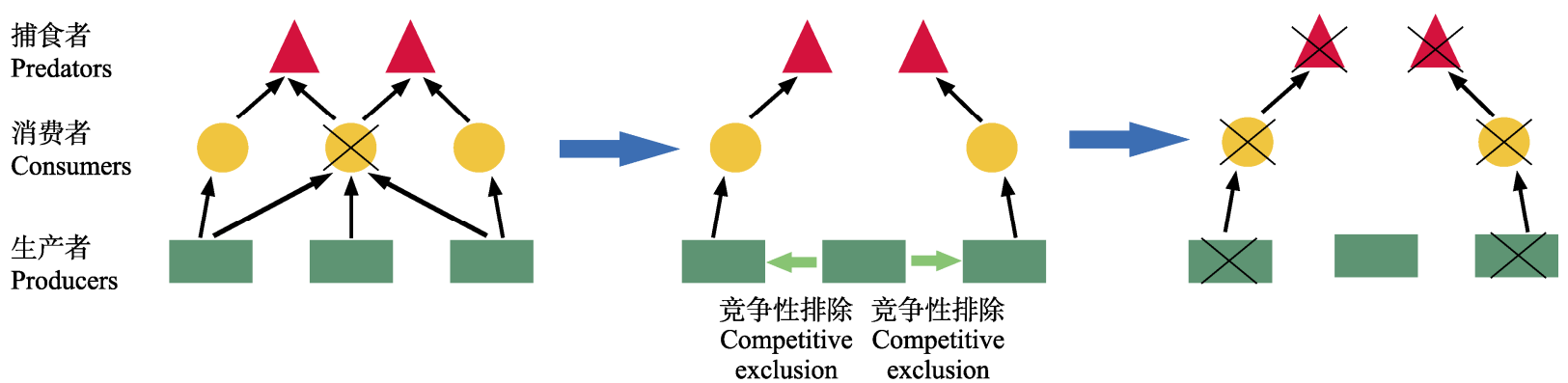

关键种为生产者

Producers as keystone species

捕食者

Predators

消费者

Consumers

生产者

Producers
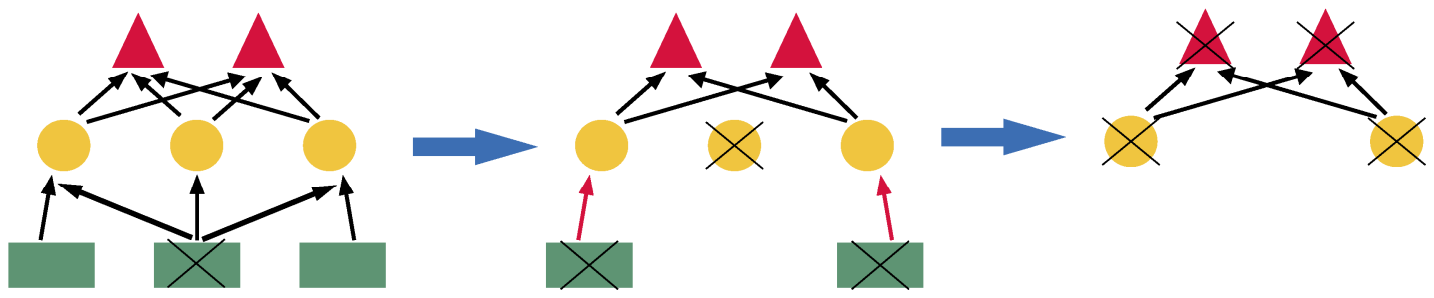

图1 关键种丧失影响食物网结构示意图。黑色箭头表示营养级关系; 红色箭头表示消费者取食力度增强。关键顶级捕食者 的缺失引起生态系统下行调控机制丧失, 刺激消费者密度快速增加从而加大对生产者的取食力度, 最终导致整个食物网崩溃; 关键消费者的丧失可能阻碍营养级间的正常能量流动，抑制捕食者的能量来源，同时改变表观竞争格局，导致生产者之间竞 争加剧, 物种数减少; 关键生产者的丧失能够增强消费者取食其他生产者的力度, 造成生产者大量灭绝, 最终威胁消费者和 顶级捕食者的生存。

Fig. 1 Schematic diagram of the impacts of loss of keystone species on food web structure. The black arrows represent trophic relationships, and the red arrows indicate the strengthened consumption by consumers. Loss of key top predators causes the absence of top-down regulation, drastically increasing consumer density and feeding intensity on producers and consequently leading to meltdown of food webs. Local extinction of key consumers may restrict energy flow between trophic levels, detrimentally affecting top predators with a simultaneous consequence of aggravating inter-specific competition among producers, which can reduce the species richness of producers. Disappearance of key producers intensifies the feeding on the remnant producers, causing the extinction of these species and in turn threatening the existence of consumers and top predators.

下降 $42 \%-47 \%$ (Bracken \& Low, 2012)。因此, 为了 确定关键生产者，需在了解食物网结构的基础上， 进一步量化分析食物网中各连接的强度。现有研究 表明陆地与水生生态系统中分解者多样性的丧失显 著降低碳氮循环速率, 影响生态系统物质能量流动 (Handa et al., 2014), 但是目前仍不明确关键分解者 丧失对食物网结构的影响。

关键种丧失对食物网结构与稳定性造成何种程 度的影响与食物网复杂性有关。在复杂食物网中往
往很少存在关键种, 因为在这样的食物网中很少存 在不可替代的物种。因而即便某一营养级中物种的 多样性发生变化, 其效应可能仅能传递至相邻营养 级(Scherber et al., 2010)。据此推测在高纬度高海拔 地区，由于本地生态系统中食物网结构简单，关键 种丧失将造成较为严重的影响。此外，食物网结构 形成的生态与进化机制目前仍有待研究。目前认为 不同营养级间的互作物种可能存在进化军备竞赛 (表1), 例如菜粉蝶(Pieris rapae)与其宿主植物拟南 
芥(Arabidopsis thaliana)在细胞色素P450基因家族 与谷胱甘肽转移酶等涉及植物防御与动物解毒功能 的基因在进化上存在显著相关性, 这在一定程度上 解释了消费者与生产者间相互适应的方式(Nallu et al., 2017)。了解这种进化方式的起源与历程, 有 助于揭示关键种的形成机制并预测其丧失带来的长 期效应。

\section{3 生物入侵的影响}

生物入侵(biological invasion)是一个物种从原 产地扩散到新环境后成功适应新环境并破坏本地生 态系统、威胁生物多样性维持的过程 (Simberloff et al., 2013)。外来物种入侵已成为一个备受关注的 全球性环境问题, 它不仅降低生物多样性水平, 破 坏生态系统功能, 而且干扰农业生产, 威胁人类健 康(Wardle et al., 2011; Simberloff et al., 2013)。我国 是受生物入侵严重干扰的国家, 目前已查明我国外 来入侵物种数达 529 种, 分布于全国各地, 其中仅松 材线虫(Bursaphelenchus xylophilus)一种就已毁灭松 林 33 万余 $\mathrm{hm}^{2}$, 对松林生态系统造成毁灭性打击(鞠 瑞亭等, 2012)。全球变化是促进生物入侵的重要因 素, 日益频繁的国际贸易导致外来入侵物种迅速增 加, 同时人类干扰破坏本地生态系统导致生态位空 缺也为外来物种提供了可乘之机(Hulme, 2009; Petitpierre et al., 2012)。此外, 全球气候变化也导致大 量物种的分布范围快速扩张, 提高了外来物种入侵 的风险(Butchart et al., 2010)。Zettlemoyer等(2019) 发现外来物种相比于本地物种具有更强的物候可塑 性, 更容易适应日益变暖的环境, 造成生物入侵。生 物入侵能够改变种间关系造成严重生态后果, 因此 伴随着生物入侵, 入侵地食物网结构往往发生剧烈 变化(Wardle et al., 2011; 鞠瑞亭等, 2012)。

外来入侵物种对同营养级物种具有很强的竞争 优势, 这可能是由于其具有内禀优势(表1), 资源利 用效率高, 可塑性强等特点, 也可能是经历了快速 适应性进化与天敌释放过程, 或者获得互利共生生 物的协助(鞠瑞亭等, 2012)。因此在入侵地食物网中, 外来入侵物种通常没有强大的天敌且对低营养级物 种的捕食强度很高, 排除同营养级物种后将大大降 低食物网复杂性并阻碍营养与能量向高营养级流动 (Ehrenfeld, 2010; Gallardo et al., 2016)。

目前普遍认为入侵植物对食物网结构的破坏最 为严重。许多入侵植物能在入侵地获得本地传粉与
种子传播动物以及共生微生物(如根瘤菌), 在剥夺 本地植物互惠网络的同时促进其种群扩张(Traveset \& Richardson, 2014)。例如, 外来千屈菜科植物 Lythrum salicaria的花序密度远高于本地植物种类, 因而成功吸引更多的传粉昆虫为其传粉, 使传粉者 造访本地植物花序的频率至少下降了 20\% (Goodell \& Parker, 2017)。许多豆科入侵植物就在入侵地获得 了共生根瘤菌, 从而大大提高了其在新环境中的适 合度(Traveset \& Richardson, 2014)。在缺乏消费者取 食的情况下, 入侵植物通过竞争排除其他植物, 最 后甚至可能导致本地消费者与捕食者灭绝，引发整 个食物网的崩溃(Pyšek et al., 2012; Gallardo et al., 2016)(图2)。例如, 外来植物互花米草(Spartina alterniflora)入侵上海崇明东滩湿地后, 造成本地优势 植物海三棱蔍草(Scirpus mariqueter)分布区迅速缩 小, 改变了地表植物碎屑理化性质与植物根系氧气 含量, 进而影响地下微生物与底栖动物群落组成, 最终降低鸟类尤其是长距离迁徙候鸟的多样性(鞠) 瑞亭等, 2012; Tong et al., 2018)。在水生生态系统中, 大型入侵水生植物能够从根本上改变本地食物网结 构, 大大减少处于最高营养级的鱼类多度 (Gallardo et al., 2016)。外来植物入侵还可能协助更多外来物 种的成功侵入, 比如入侵松科植物与其共生菌根真 菌(Dickie et al., 2010), 加剧生物入侵对食物网的影 响, 造成入侵崩溃(表1)。此外, 入侵植物降低植物 多样性, 能够改变土壤微生物群落, 影响地下食物 网结构(Kardol \& Wardle, 2010)。

入侵捕食者或者病原体在竞争排除同营养级中 乡土种的同时可能由于其捕食偏好改变消费者表观 竞争格局, 由此产生的营养级联效应可能导致消费 者物种组成与群落结构的改变并影响低营养级物种 (图2)。外来鱼类物种入侵能够抑制底栖动物与浮游 生物数量, 从而促进浮游植物的生长(Gallardo et al., 2016)。此外，某些入侵捕食者通过捕食在不同生境 间迁徙的消费者, 从而改变本地植被组成。例如, 北 极狐(Alopex lagopus)入侵阿留申群岛的部分岛屿后 大肆捕猎海鸟, 导致海鸟数量快速下降, 由此造成 鸟粪匮乏, 使得土壤肥力下降，从而导致当地原有 的草原植被类型向适应贫痊土壤的苔原植被类型转 变(Croll et al., 2005)。然而某些广食性外来捕食者的 入侵也可能替代原有捕食者的作用，通过下行调控 维持食物网稳定。例如, 在澳大利亚干旱地区, 移除 


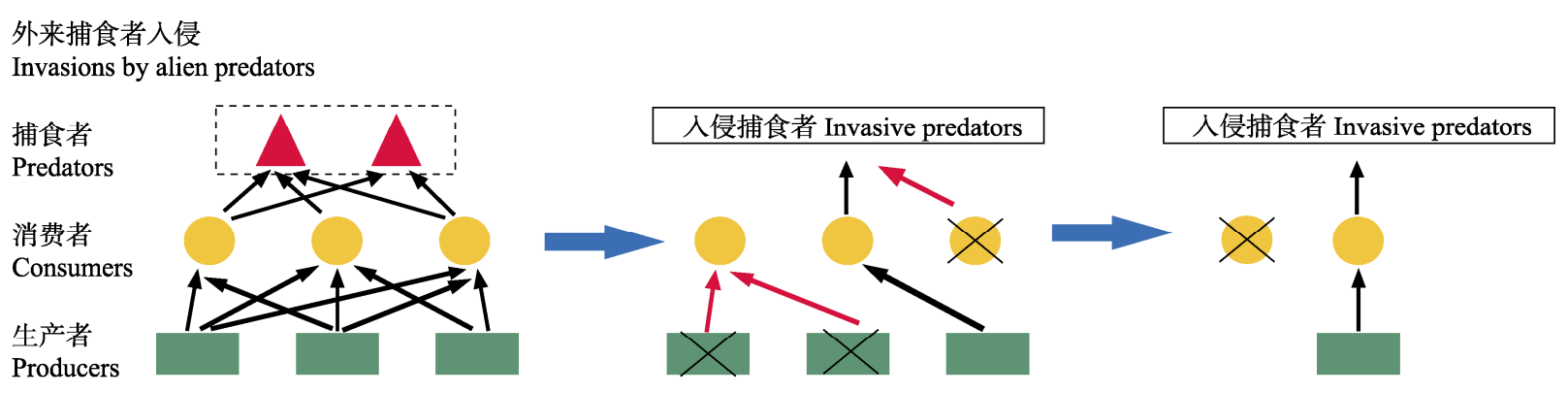

\section{外来消费者入侵}

Invasions by alien consumers

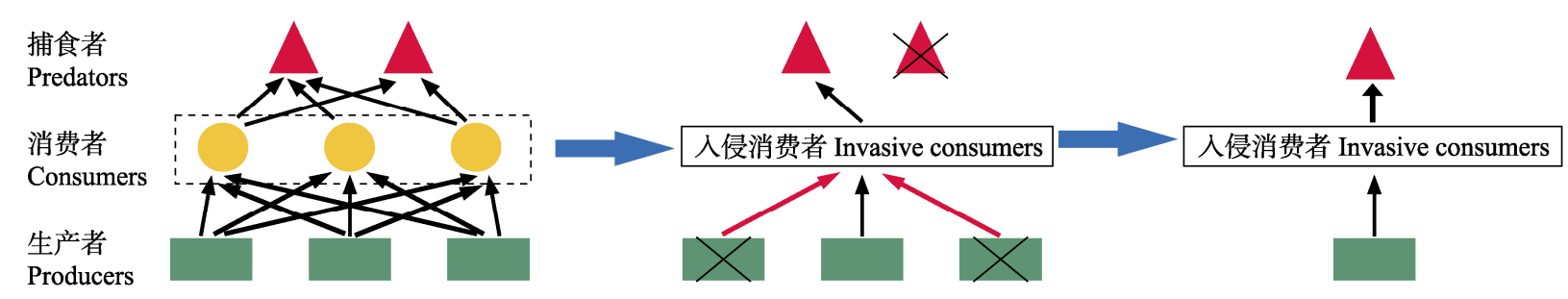

外来生产者入侵

Invasions by alien producers

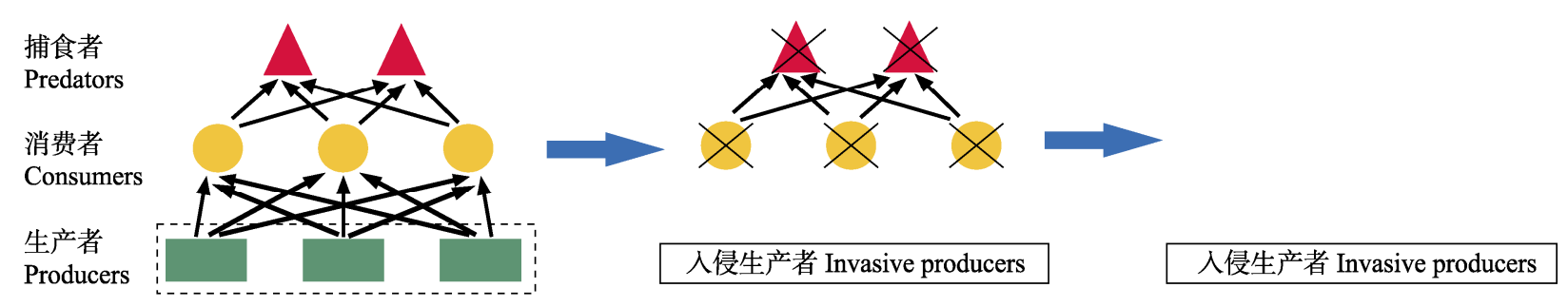

图2 外来物种入侵影响食物网结构示意图。黑色箭头表示营养级关系; 红色箭头表示消费者取食力度或捕食者捕食强度增 强。入侵捕食者在竞争排除同营养级中乡土种的同时由于其捕食偏好改变消费者表观竞争格局并影响低营养级; 入侵消费者 通过竞争减少消费者物种数对生产者产生较大的取食压力，同时阻断顶级捕食者的食物来源，进而破坏食物网的上行和下行 调控, 改变食物网结构; 在缺乏消费者取食的情况下, 入侵生产者通过竞争排除其他生产者并阻断能量流动, 导致消费者与 捕食者灭绝，引发整个食物网的崩溃。

Fig. 2 Schematic diagram of the impacts of alien invasive species on food web structure. The black arrows represent trophic relationships, and the red arrows indicate the increased consumption or the strengthened predation. Invasive predators can exclude native predators by competition and alter the interspecific competition of consumer due to their feeding preference, impacting the species composition at lower trophic levels. Invasive consumers exclude native consumers, intensify feeding on some producers and restrict food resources of top predators, disturbing top-down and bottom-up regulations and changing food web structure. In the absence of natural enemies, invasive producers exclude native producers and cut off energy flow, leading to massive extinction of consumers and top predators and meltdown of food webs.

外来顶级捕食者澳洲野狗(Canis lupus dingo)致使消 费者与中型捕食者赤狐(Vulpes vulpes)的密度提高, 从而使草地盖度与小型哺乳动物种类减少(Letnic et al., 2009)。

入侵消费者与入侵杂食动物对食物网影响的研 究案例较少, 其潜在威胁可能是通过竞争排除减少 消费者物种数并对生产者产生较大的取食压力, 同 时阻断部分顶级捕食者的食物来源, 进而破坏食物 网的上行和下行调控, 改变食物网结构(图2)。例如,
Gibson等(2013)发现在泰国水库岛屿森林中，本地 小型哺乳动物由于外来啮齿动物的入侵而加速灭 绝。此外, 入侵土壤微生物可能影响地表植物获取 根瘤菌, 从而影响植物多样性以及食物网结构 (Ehrenfeld, 2010; Kardol \&Wardle, 2010)。

掌握生物入侵破坏食物网结构的机制, 对生态 修复具有重大应用价值。尽管清除入侵物种是开展 生态修复的基础，但是针对不同入侵物种在对其进 行清除后，需开展不同的后续恢复措施。例如移除

www.plant-ecology.com 
入侵植物后应该尽量恢复本地动植物群落, 通过占 据生态位预防入侵植物再次侵袭的同时, 促进地下 生物群落的恢复; 而消灭入侵捕食者后则需同时恢 复本地顶级捕食者与消费者类群, 以确保营养级联 效应与食物网下行调控的力度(Kardol \& Wardle, 2010)。

\section{2 讨论和展望}

种间关系的稳定是维持食物网中上行与下行调 控的基础, 也是促使物种共存与生物多样性的重要 机制(Terborgh, 2015; Wang et al., 2019a, 2019b, 2020)。 人类活动导致的全球变化除了能够直接导致物种灭 绝, 还能通过改变种间关系破坏食物网结构, 威胁 生物多样性的长期维持(Bascompte, 2009)。由上文 所述, 全球变化造成物候错配改变不同营养级物种 的互作关系强度, 影响物质能量流动与食物网调控, 进而改变食物网结构; 同时全球变化导致食物网关 键种丧失能够造成营养级间捕食关系发生变化甚至 缺失, 致使物种遭遇过度捕食而灭绝, 或者打破同 一营养级中的种间竞争平衡而降低物种多样性; 此 外, 入侵物种通过竞争排除同营养级物种阻断食物 网中物质能量流动, 降低食物网结构复杂性。不同 生态系统对上述三种干扰机制的响应可能与食物网 中互作物种间协同进化历程、种间关系的专一性程 度以及食物网内部的嵌套结构有关。

种间关系的维持往往伴随着互作物种间长期的 协同进化, 通常这种进化关系越紧密种间关系越牢 固, 受外界干扰而发生快速变化的可能性就较低; 而协同进化关系不紧密的互作物种在全球变化背景 下更容易发生种间关系变化(例如种间互作强度变 化、互惠关系向拮抗关系转变等), 进而导致食物网 结构迅速改变(Futuyma \& Agrawal, 2009; Kiers et al., 2010)。然而, 具有紧密协同进化关系的物种一般为 专性物种(specialist), 双方任何一方灭绝经常会导 致另一方也灭绝, 因此在全球变化干扰强度大的情 况下与泛性物种 (generalist) 相比具有较高的灭绝风 险(Weiner et al., 2014)(图1, 图2)。此外, 在复杂食物 网中并非所有物种均与其他任一物种间存在种间关 系, 往往形成与部分物种间互作关系密切而与其他 物种间关系较为疏远的情况, 即形成了嵌套结构 (Dalsgaard et al., 2013)。目前认为嵌套结构的存在能 够有效阻止种间关系变化产生的影响蔓延至整个食
物网, 因而促进群落稳定性和物种共存(Neutel et al., 2007)。

总而言之, 全球变化对食物网结构产生影响的实 质是物种是否能够适应日益变化的生态环境, 形成生 态-进化动态(eco-evolutionary dynamics)(Hoffmann \& Sgrò, 2011; Zuppinger-Dingley et al., 2014)。同时种 间关系与食物网结构受全球变化的影响程度主要取 决于维系互作关系的关键性状是否受到变化环境的 选择(Althoff et al., 2014)。然而, 目前在大多数生态 系统中对食物网物种组成与结构仍未有清晰认识, 并且物种适应生物与非生物环境的机制研究也尚处 于初始阶段。因此, 今后研究的重点应聚焦于生态 网络分析尤其是地上-地下生物与植物-微生物的相 互作用(Bardgett \& van der Putten, 2014; Traveset \& Richardson, 2014), 揭示完整食物网结构并全面评 估全球变化造成的影响; 同时还应利用日益成熟的 全基因组测序、表观遗传学分析与基因组关联分析 技术，确定调控物种关键适应性状特征的基因 (Olsen et al., 2016; Nallu et al., 2017), 建立基因组性状-环境因子动态数据库, 厘清全球变化背景下 种间关系与食物网结构改变的机制, 以预测未来生 物多样性变化的趋势并制定合理的生物多样性保护 与生态修复措施。

\section{参考文献}

Althoff DM, Segraves KA, Johnson MTJ (2014). Testing for coevolutionary diversification: linking pattern with process. Trends in Ecology \& Evolution, 29, 82-89.

Bardgett RD, van der Putten WH (2014). Belowground biodiversity and ecosystem functioning. Nature, 515, 505-511.

Bascompte J (2009). Disentangling the web of life. Science, 325, 416-419.

Both C, van Asch M, Bijlsma RG, van den Burg AB, Visser ME (2009). Climate change and unequal phenological changes across four trophic levels: constraints or adaptations? Journal of Animal Ecology, 78, 73-83.

Both C, van Turnhout CAM, Bijlsma RG, Siepel H, van Strien AJ, Foppen RPB (2010). Avian population consequences of climate change are most severe for long-distance migrants in seasonal habitats. Proceedings of the Royal Society B: Biological Sciences, 277, 1259-1266.

Bracken MES, Low NHN (2012). Realistic losses of rare species disproportionately impact higher trophic levels. Ecology Letters, 15, 461-467.

Butchart SHM, Walpole M, Collen B, van Strien AJ, Scharlemann JPW, Almond REA, Baillie JEM, Bomhard B, 
Brown C, Bruno J, Carpenter KE, Carr GM, Chanson J, Chenery AM, Csirke J, et al. (2010). Global biodiversity: indicators of recent declines. Science, 328, 1164-1168.

Cardinale BJ, Duffy JE, Gonzalez A, Hooper DU, Perrings C, Venail P, Narwani A, Mace GM, Tilman D, Wardle DA, Kinzig AP, Daily GC, Loreau M, Grace JB, Larigauderie A, Srivastava DS, Naeem S (2012). Biodiversity loss and its impact on humanity. Nature, 486, 59-67.

Croll DA, Maron JL, Estes JA, Danner EM, Byrd GV (2005). Introduced predators transform subarctic islands from grassland to tundra. Science, 307, 1959-1961.

Dalsgaard B, Trøjelsgaard K, Martín González AM, NoguésBravo D, Ollerton J, Petanidou T, Sandel B, Schleuning M, Wang ZH, Rahbek C, Sutherland WJ, Svenning JC, Olesen JM (2013). Historical climate-change influences modularity and nestedness of pollination networks. Ecography, 36, 1331-1340.

Dickie IA, Bolstridge N, Cooper JA, Peltzer DA (2010). Co-invasion by Pinus and its mycorrhizal fungi. New Phytologist, 187, 475-484.

Dunne JA, Williams RJ, Martinez ND (2002). Food-web structure and network theory: the role of connectance and size. Proceedings of the National Academy of Sciences of the United States of America, 99, 12917-12922.

Ehrenfeld JG (2010). Ecosystem consequences of biological invasions. Annual Review of Ecology, Evolution, and Systematics, 41, 59-80.

Elton CS (1958). The Ecology of Invasions by Animals and Plants. Springer, Boston.

Estes JA, Terborgh J, Brashares JS, Power ME, Berger J, Bond WJ, Carpenter SR, Essington TE, Holt RD, Jackson JBC, Marquis RJ, Oksanen L, Oksanen T, Paine RT, Pikitch EK, et al. (2011). Trophic downgrading of planet earth. Science, 333, 301-306.

Ewers RM, Didham RK (2006). Confounding factors in the detection of species responses to habitat fragmentation. Biological Reviews, 81, 117-142.

Futuyma DJ, Agrawal AA (2009). Macroevolution and the biological diversity of plants and herbivores. Proceedings of the National Academy of Sciences of the United States of America, 106, 18054-18061.

Gallardo B, Clavero M, Sánchez MI, Vilà M (2016). Global ecological impacts of invasive species in aquatic ecosystems. Global Change Biology, 22, 151-163.

Gibson L, Lynam AJ, Bradshaw CJA, He FL, Bickford DP, Woodruff DS, Bumrungsri S, Laurance WF (2013). Near-complete extinction of native small mammal fauna 25 years after forest fragmentation. Science, 341, 1508-1510.

Gilman SE, Urban MC, Tewksbury J, Gilchrist GW, Holt RD (2010). A framework for community interactions under climate change. Trends in Ecology \& Evolution, 25,
325-331.

Goodell K, Parker IM (2017). Invasion of a dominant floral resource: effects on the floral community and pollination of native plants. Ecology, 98, 57-69.

Haddad NM, Brudvig LA, Clobert J, Davies KF, Gonzalez A, Holt RD, Lovejoy TE, Sexton JO, Austin MP, Collins CD (2015). Habitat fragmentation and its lasting impact on earth's ecosystems. Science Advances, 1, e1500052. DOI: 10.1126/sciadv.1500052.

Hairston NG, Smith FE, Slobodkin LB (1960). Community structure, population control, and competition. The American Naturalist, 94, 421-425.

Handa IT, Aerts R, Berendse F, Berg MP, Bruder A, Butenschoen O, Chauvet E, Gessner MO, Jabiol J, Makkonen M, McKie BG, Malmqvist B, Peeters ETHM, Scheu S, Schmid B, et al. (2014). Consequences of biodiversity loss for litter decomposition across biomes. Nature, 509, 218-221.

Hansson LA, Nicolle A, Granéli W, Hallgren P, Kritzberg E, Persson A, Björk J, Nilsson PA, Brönmark C (2013). Food-chain length alters community responses to global change in aquatic systems. Nature Climate Change, 3, 228-233.

Hebert PDN, Cywinska A, Ball SL, DeWaard JR (2003). Biological identifications through DNA barcodes. Proceedings of the Royal Society B: Biological Sciences, 270, 313-321.

Hoffmann AA, Sgrò CM (2011). Climate change and evolutionary adaptation. Nature, 470, 479-485.

Hughes BB, Eby R, van Dyke E, Tinker MT, Marks CI, Johnson KS, Wasson K (2013). Recovery of a top predator mediates negative eutrophic effects on seagrass. Proceedings of the National Academy of Sciences of the United States of America, 110, 15313-15318.

Hulme PE (2009). Trade, transport and trouble: managing invasive species pathways in an era of globalization. Journal of Applied Ecology, 46, 10-18.

Ju RT, Li H, Shi ZR, Li B (2012). Progress of biological invasions research in China over the last decade. Biodiversity Science, 20, 581-611. [鞠瑞亭, 李慧, 石正人, 李博 (2012). 近十年中国生物入侵研究进展. 生物多样性, 20, 581-611.]

Kadoya T, Gellner G, McCann KS (2018). Potential oscillators and keystone modules in food webs. Ecology Letters, 21, 1330-1340.

Kardol P, Wardle DA (2010). How understanding aboveground belowground linkages can assist restoration ecology. Trends in Ecology \& Evolution, 25, 670-679.

Kiers TE, Palmer TM, Ives AR, Bruno JF, Bronstein JL (2010). Mutualisms in a changing world: an evolutionary perspective. Ecology Letters, 13, 1459-1474.

Letnic M, Koch F, Gordon C, Crowther MS, Dickman CR (2009). Keystone effects of an alien top-predator stem

www.plant-ecology.com 
extinctions of native mammals. Proceedings of the Royal Society B: Biological Sciences, 276, 3249-3256.

Li Y, Li GY, Mu JP, Sun SC (2008). Effects of consumer diversity on food web structure and ecosystem functioning: current knowledge and perspectives. Acta Ecologica Sini$c a, 28,388-398$. [李妍, 李国勇, 慕军鹏, 孙书存 (2008). 消费者多样性对食物网结构和生态系统功能的 影响. 生态学报, 28, 388-398.]

Lindeman RL (1942). The trophic-dynamic aspect of ecology. Ecology, 23, 399-418.

Liu YZ, Reich PB, Li GY, Sun SC (2011). Shifting phenology and abundance under experimental warming alters trophic relationships and plant reproductive capacity. Ecology, 92, 1201-1207.

May RM (1972). Will a large complex system be stable? Nature, 238, 413-414.

Memmott J, Waser NM, Price MV (2004). Tolerance of pollination networks to species extinctions. Proceedings of the Royal Society B: Biological Sciences, 271, 2605-2611.

Murphy GEP, Romanuk TN (2014). A meta-analysis of declines in local species richness from human disturbances. Ecology and Evolution, 4, 91-103.

Nallu S, Hill J, Don K, Sahagun C, Zhang W, Meslin C, Snell-Rood E, Clark NL, Morehouse NI, Bergelson J, Wheat CW, Kronforst MR (2017). The molecular genetic basis of herbivory between butterflies and their host-plants. Nature Ecology and Evolution, 2, 1418-1427.

Neutel AM, Heesterbeek JAP, van de Koppel J, Hoenderboom G, Vos A, Kaldeway C, Berendse F, de Ruiter PC (2007). Reconciling complexity with stability in naturally assembling food webs. Nature, 449, 599-602.

Olsen JL, Rouze P, Verhelst B, Lin Y, Bayer T, Collen J, Dattolo E, de Paoli E, Dittami SM, Maumus F (2016). The genome of the seagrass Zostera marina reveals angiosperm adaptation to the sea. Nature, 530, 331-335.

Petitpierre B, Kueffer C, Broennimann O, Randin C, Daehler C, Guisan A (2012). Climatic niche shifts are rare among terrestrial plant invaders. Science, 335, 1344-1348.

Pimm SL (1979). The structure of food webs. Theoretical Population Biology, 16, 144-158.

Potts SG, Biesmeijer JC, Kremen C, Neumann P, Schweiger O, Kunin WE (2010). Global pollinator declines: trends, impacts and drivers. Trends in Ecology \& Evolution, 25, 345-353.

Pyšek P, Jarošík V, Hulme PE, Pergl J, Hejda M, Schaffner U, Vilà $M$ (2012). A global assessment of invasive plant impacts on resident species, communities and ecosystems: the interaction of impact measures, invading species' traits and environment. Global Change Biology, 18, 1725-1737.

Ricciardi A, MacIsaac HJ (2000). Recent mass invasion of the North American Great Lakes by Ponto-Caspian species. Trends in Ecology \& Evolution, 15, 62-65.
Ripple WJ, Estes JA, Beschta RL, Wilmers CC, Ritchie EG, Hebblewhite M, Berger J, Elmhagen B, Letnic M, Nelson MP, Schmitz OJ, Smith DW, Wallach AD, Wirsing AJ (2014). Status and ecological effects of the world's largest carnivores. Science, 343, 1241484. DOI: 10.1126/science. 1241484.

Ripple WJ, Newsome TM, Wolf C, Dirzo R, Everatt KT, Galetti M, Hayward MW, Kerley GIH, Levi T, Lindsey PA, MacDonald DW, Malhi Y, Painter LE, Sandom CJ, Terborgh J, van Valkenburgh B (2015). Collapse of the world's largest herbivores. Science Advances, 1, e1400103. DOI: 10.1126/sciadv.1400103.

Scherber C, Eisenhauer N, Weisser WW, Schmid B, Voigt W, Fischer M, Schulze ED, Roscher C, Weigelt A, Allan E, Besler H, Bonkowski M, Buchmann N, Buscot F, Clement LW, et al. (2010). Bottom-up effects of plant diversity on multitrophic interactions in a biodiversity experiment. $\mathrm{Na}$ ture, 468, 553-556.

Simberloff D, Martin JL, Genovesi P, Maris V, Wardle DA, Aronson J, Courchamp F, Galil BS, García-Berthou E, Pascal M, Pyšek P, Sousa R, Tabacchi E, Vilà M (2013). Impacts of biological invasions: What's what and the way forward. Trends in Ecology \& Evolution, 28, 58-66.

Sydeman WJ, Poloczanska E, Reed TE, Thompson SA (2015). Climate change and marine vertebrates. Science, 350, 772-777.

Tao J, He DK, Kennard MJ, Ding CZ, Bunn SE, Liu CL, Jia YT, Che RX, Chen YF (2018). Strong evidence for changing fish reproductive phenology under climate warming on the Tibetan Plateau. Global Change Biology, 24, 2093-2104.

Taubert F, Fischer R, Groeneveld J, Lehmann S, Muller MS, Rodig E, Wiegand T, Huth A (2018). Global patterns of tropical forest fragmentation. Nature, 554, 519-522.

Terborgh J, Lopez L, Nuñez P, Rao M, Shahabuddin G, Orihuela G, Riveros M, Ascanio R, Adler GH, Lambert TD, Balbas L (2001). Ecological meltdown in predator-free forest fragments. Science, 294, 1923-1926.

Terborgh JW (2015). Toward a trophic theory of species diversity. Proceedings of the National Academy of Sciences of the United States of America, 112, 11415-11422.

Thackeray SJ, Henrys PA, Hemming D, Bell JR, Botham MS, Burthe SJ, Helaouet P, Johns DG, Jones ID, Leech DI (2016). Phenological sensitivity to climate across taxa and trophic levels. Nature, 535, 241-245.

Tong X, Wang R, Chen XY (2018). Expansion or invasion? A response to Nackley et al. Trends in Ecology \& Evolution, 33, 234-235.

Traveset A, Richardson DM (2014). Mutualistic interactions and biological invasions. Annual Review of Ecology, Evolution, and Systematics, 45, 89-113.

Trisos CH, Merow C, Pigot AL (2020). The projected timing of 
abrupt ecological disruption from climate change. Nature, 580, 496-501.

Tylianakis JM, Didham RK, Bascompte J, Wardle DA (2008). Global change and species interactions in terrestrial ecosystems. Ecology Letters, 11, 1351-1363.

Wabnitz C, Balazs G, Beavers S, Bjorndal KA, Bolten AB, Christensen V, Hargrove S, Pauly D (2010). Ecosystem structure and processes at Kaloko Honokohau, focusing on the role of herbivores, including the green sea turtle Chelonia mydas, in reef resilience. Marine Ecology Progress Series, 420, 27-44.

Wang R, Chen XY, Chen Y, Wang G, Dunn DW, Quinnell RJ, Compton SG (2019a). Loss of top-down biotic interactions changes the relative benefits for obligate mutualists. Proceedings of the Royal Society B: Biological Sciences, 286, 20182501. DOI: 10.1098/rspb.2018.2501.

Wang R, Shi YS, Zhang YX, Xu GF, Shen GC, Chen XY (2019b). Distance-dependent seed-seedling transition in the tree Castanopsis sclerophylla is altered by fragment size. Communications Biology, 2, 277. DOI: 10.1038/s42003019-0528-x.

Wang R, Zhang X, Shi YS, Li YY, Wu JG, He FL, Chen XY (2020). Habitat fragmentation changes top-down and bottom-up controls of food webs. Ecology, 101, e03062. DOI: 10.1002/ecy.3062.

Wang SP, Brose U (2018). Biodiversity and ecosystem functioning in food webs: the vertical diversity hypothesis. Ecology Letters, 21, 9-20.

Wang SP, Brose U, Gravel D (2019c). Intraguild predation enhances biodiversity and functioning in complex food webs. Ecology, 100, e02616. DOI: 10.1002/ecy.2616.

Wang YY, Xu J, Lei GC (2013). Proximate and ultimate determinants of food chain length. Acta Ecologica Sinica, 33, 5990-5996. [王玉玉, 徐军, 雷光春 (2013). 食物链长度 远因与近因研究进展综述. 生态学报, 33, 5990-5996.]

Wardle DA, Bardgett RD, Callaway RM, der Putten WHV (2011). Terrestrial ecosystem responses to species gains and losses. Science, 332, 1273-1277.

Weiner CN, Werner M, Linsenmair KE, Blüthgen N (2014). Land-use impacts on plant-pollinator networks: interaction strength and specialization predict pollinator declines. Ecology, 95, 466-474.

Wilson MC, Chen XY, Corlett RT, Didham RK, Ding P, Holt RD, Holyoak M, Hu G, Hughes AC, Jiang L, Laurance WF, Liu JJ, Pimm SL, Robinson SK, Russo SE, Si XF, Wilcove DS, Wu JG, Yu MJ (2016). Habitat fragmentation and biodiversity conservation: key findings and future challenges. Landscape Ecology, 31, 219-227.

You MS, Yue Z, He WY, Yang XH, Yang G, Xie M, Zhan DL, Baxter SW, Vasseur L, Gurr GM, Douglas CJ, Bai JL, Wang P, Cui K, Huang SG, et al. (2013). A heterozygous moth genome provides insights into herbivory and detoxification. Nature Genetics, 45, 220-225.

Zander A, Bersier L, Gray SM (2017). Effects of temperature variability on community structure in a natural microbial food web. Global Change Biology, 23, 56-67.

Zettlemoyer MA, Schultheis EH, Lau JA (2019). Phenology in a warming world: differences between native and nonnative plant species. Ecology Letters, 22, 1253-1263.

Zhao L, Zhang HY, O'Gorman EJ, Tian W, Ma A, Moore JC, Borrett SR, Woodward G (2016). Weighting and indirect effects identify keystone species in food webs. Ecology Letters, 19, 1032-1040.

Zou JW, Rogers WE, Siemann E (2007). Differences in morphological and physiological traits between native and invasive populations of Sapium sebiferum. Functional Ecology, 21, 721-730.

Zuppinger-Dingley D, Schmid B, Petermann JS, Yadav V, de Deyn GB, Flynn DFB (2014). Selection for niche differentiation in plant communities increases biodiversity effects. Nature, 515, 108-111. 